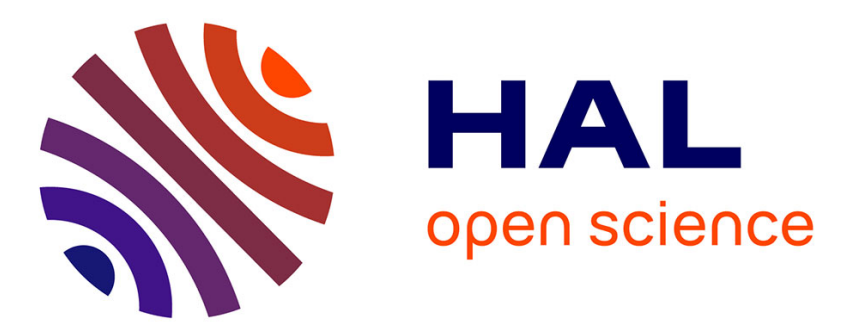

\title{
Stability Robustness in the presence of exponentially unstable isolated equilibria
}

\author{
David Angeli, Laurent Praly
}

\section{To cite this version:}

David Angeli, Laurent Praly. Stability Robustness in the presence of exponentially unstable isolated equilibria. 49th IEEE Conference on Decision and Control, Dec 2010, Atlanta, United States. pp.15811586, 10.1109/CDC.2010.5717582 . hal-00554432

HAL Id: hal-00554432

https://hal-mines-paristech.archives-ouvertes.fr/hal-00554432

Submitted on 10 Jan 2011

HAL is a multi-disciplinary open access archive for the deposit and dissemination of scientific research documents, whether they are published or not. The documents may come from teaching and research institutions in France or abroad, or from public or private research centers.
L'archive ouverte pluridisciplinaire HAL, est destinée au dépôt et à la diffusion de documents scientifiques de niveau recherche, publiés ou non, émanant des établissements d'enseignement et de recherche français ou étrangers, des laboratoires publics ou privés. 


\section{Stability Robustness in the presence of exponentially unstable isolated equilibria}

\author{
David Angeli \\ Imperial College, London, U.K. \\ University of Firenze, Italy
}

\author{
Laurent Praly \\ Centre Automatique et Systèmes \\ Ecole des Mines de Paris, France
}

\begin{abstract}
This note studies nonlinear systems evolving on manifolds with a finite number of asymptotically stable equilibria and a Lyapunov function which strictly decreases outside equilibrium points. If the linearizations at unstable equilibria have at least one eigenvalue with positive real part, then almost global asymptotic stability turns out to be robust with respect to sufficiently small disturbances in the $L_{\infty}$ norm. Applications of this result are shown in the study of almost global Input-toState stability.
\end{abstract}

\section{INTRODUCTION AND MOTIVATIONS}

Stability notions with respect to exogenous signals are a key tool in nonlinear control. On one hand they allow to analyze stability of interconnected systems in terms of InputOutput gains of individual subsystems, see for instance [6]. On the other, they provide quantitative estimates of how the system reacts to exogenous disturbances. Two approaches have been particularly fruitful, both in theory and practice. These are the so-called $H_{\infty}$ and Input-to-State Stability frameworks, [16], [13]. Both approaches extend the classical Lyapunov method, traditionally used to establish internal stability properties, to systems with inputs and outputs. Indeed, in analogy to the classical Lyapunov method, they exploit state-space descriptions of system's dynamics and energylike functions in order to asses the stability and robustness of a system with respect to internal and external perturbations. The theory is very developed for nonlinear systems which are defined on Euclidean spaces and with a globally asymptotically stable equilibrium point. However, applicability to more general set-ups is not straightforward. For instance smooth systems evolving on manifolds or systems whose attractor is something more complicated than a single equilibrium are not suitable to a global approach by using these analytical tools. As pointed out in [1], topological obstructions to global stability arise even in the absence of exogenous disturbances. One natural way to relax global requirements is therefore to consider almost global stability notions with respect to a single equilibrium or, more generally, to the non-trivial attractor of interest (for instance multiple equilibria). This entails a deep revision of the analytical techniques involved.

An attempt in this direction was discussed in [1], with a new definition of almost global Input-to-State Stability (aISS) and the proposition of some analytical techniques which may be employed to establish aISS for non-trivial examples of nonlinear systems. The main result in [1] makes use of the so called density functions, which were recently introduced by Rantzer as a natural dual to Lyapunov functions, in the study of almost global stability and attractivity notions, [11], [12]. While software tools to automatically find density functions for certain classes of systems are beginning to become available, [10], [9], recent analysis has also highlighted that explicit closed-form expressions of smooth dual Lyapunov functions in the case of systems with saddle points of negative divergence, [2], might actually not exist in most cases.

The difficulties in finding such functions pushed the authors in the direction of proposing a complementary set of tools for the study of stability robustness in the presence of unstable and antistable invariant sets. The techniques heavily rely on the stable and unstable manifolds theory of dynamical systems, in particular on their time-varying adaptations. This paper was motivated by an open problem publicly posed by one of the authors during the 2009 Oberwolfach meeting in Control Theory, [3], and provides together with a positive answer to the question thereby formulated, a result to address similar questions in general and realistic scenarios.

Just as a motivating example, which will later be discussed in more detail, we recall the question posed in [3]. The system under consideration is a pendulum with friction, of equations

$$
\begin{aligned}
\dot{\theta} & =\omega \\
\dot{\omega} & =-\sin (\theta)-\omega+d,
\end{aligned}
$$

whose state variable $x=[\theta, \omega]$ takes values in the manifold $\mathbb{S} \times \mathbb{R}$. For $d=0$, that is in the absence of exogenous torque disturbances, it is well-known that almost all solutions will converge to the equilibrium $[0,0]$, corresponding to the pendulum pointing downwards. On the other hand, the upright position of the pendulum $[\pi, 0]$ is an hyperbolic saddle point of negative divergence (divergence is equal to -1 everywhere in state space). Therefore, a zero-measure set of initial conditions (in particular those belonging to the socalled stable manifold which is in this case one-dimensional) give rise to solutions asymptotically approaching the upright equilibrium. One might thus wonder whether, in the presence of non-zero disturbances, almost all initial conditions give rise to solutions which are ultimately bounded within some neighborhood of the downwards equilibrium. In particular, for this to happen with continuity, one should ask that the amplitude of such neighborhood be bounded from above in terms of the $L_{\infty}$ norm of the disturbance, modulated by some 
$\mathcal{K}$ function ${ }^{1}$.

The answer to this question is positive and follows by applying our Main Result. This is a connection between existence of Lyapunov functions with strictly negative derivative and robustness of almost global asymptotic stability to exogenous disturbances of sufficiently small amplitude. While such results are by now well-known and frequently quoted in the case of GAS (see for instance the converse Lyapunov theorems provided in [15]), rather different techniques are needed for almost global stability analysis. Combined with more standard tools for ultimate boundedness or practical Input-to-State Stability analysis, the technique configures a separation principle for claiming almost global Input-to-State Stability.

\section{Problem formulation AND Main Result}

This note deals with nonlinear systems of the following form:

$$
\dot{x}(t)=f(x(t), d(t))
$$

with state $x$ taking value in some $n$-dimensional $\mathcal{C}^{2}$ connected, orientable, Riemannian manifold $M$ without boundary, $f: M \times D \rightarrow T_{x} M$ of class $\mathcal{C}^{1}$-Lipschitz and $D$ a closed set of $\mathbb{R}^{m}$. We denote by $X\left(t, x, t_{0} ; d\right)$ its solution which is at $x$ at time $t_{0}$ and we call unperturbed system the following :

$$
\dot{x}(t)=f(x(t), 0) \doteq f_{0}(x(t)) .
$$

We assume :

1) existence of a nonnegative and proper $\mathcal{C}^{1}$ function $V$ : $M \rightarrow \mathbb{R}$ such that we have ${ }^{2}:$

$$
\left.L_{f} V\right|_{x, 0}<0 \quad \forall x \in M: f_{0}(x) \neq 0
$$

2) any equilibrium $x_{\ell}$ which is not asymptotically stable, is isolated and such that at least one eigenvalue of $d f_{0}\left(x_{\ell}\right): T_{x_{\ell}} M \rightarrow T_{x_{\ell}} M$ has strictly positive real part, where $d f_{0}(x)$ denotes the differential of $f_{0}$ at $x$.

Notice that (4) implies that stationary points of $V$ are equilibria (the converse need not be true). Also, asymptotically stable equilibria are, by definition, necessarily isolated and with an open basin of attraction.

If $M$ is a not compact, let $v$ be a real number arbitrary up to the fact that the compact set

$$
\mathfrak{C}=\{x: V(x) \leq v\}
$$

contains at least one asymptotically stable equilibrium and no equilibrium on its boundary. If $M$ is compact, we let :

$$
\mathfrak{C}=M \text {. }
$$

Since equilibria of the undisturbed system are isolated, $\mathfrak{C}$ contains a finite number $\mathfrak{L}$ of them which we denote by $x_{\ell}$ with $\ell$ ranging in $\{1,2, \ldots, \mathfrak{L}\}$. Also, we denote by $E_{s}$ the finite set of those which are asymptotically stable.

\footnotetext{
${ }^{1} \mathrm{~A}$ function $\gamma:[0,+\infty) \rightarrow[0,+\infty)$ is of class $\mathcal{K}$ if continuous, increasing and $\gamma(0)=0$.

${ }^{2}$ We use the notation $\left.L_{f} V\right|_{x, d}$ to denote the Lie derivative of $V$ along $f$ at a point $x$ when the perturbation is $d$.
}

Proposition 1: Under the above assumptions, there exist a real number $\Delta>0$ and a class $\mathcal{K}$ function $\gamma$ such that, for each measurable perturbation $d: \mathbb{R} \rightarrow D \subset \mathbb{R}^{m}$ with $L^{\infty}$ norm smaller than $\Delta$, and for each $t_{0}$ in $\mathbb{R}$, there exists a set $\mathfrak{B}_{d}\left(t_{0}\right) \subset M$ of zero Riemannian volume such that, every solution $X\left(t, x, t_{0} ; d\right)$ of (2) with $x$ in $\mathfrak{C} \backslash \mathfrak{B}_{d}\left(t_{0}\right)$ is defined at least on $\left[t_{0},+\infty\right)$ and satisfies :

$$
\lim _{t \rightarrow+\infty} \mathfrak{d}_{M}\left(X\left(t, x, t_{0} ; d\right), E_{s}\right) \leq \lim _{t \rightarrow+\infty} \text { ess. } \sup _{s \geq t} \gamma(|d(s)|)
$$

where $\mathfrak{d}_{M}(x, y)$ denotes the Riemannian distance between $x$ and $y$ in $M$.

To prove this Proposition, we shall need the following Lemma whose proof is not included for space reasons. It relies heavily on the results in [4] and is basically a variant of the Main Result therein discussed.

Lemma 1: Let $x_{\ell}$ be an isolated equilibrium of the unperturbed system (3) such that at least one eigenvalue of $d f_{0}\left(x_{\ell}\right)$ has strictly positive real part. There exist a neighborhood $\mathcal{P}\left(x_{\ell}\right)$ of $x_{\ell}$, a strictly positive real number $D_{p}\left(x_{\ell}\right)$, a nonnegative integer $p<n$ and a bounded open set $\mathfrak{O}_{\ell}$ in $\mathbb{R}^{p}$, such that, for each measurable perturbation $d: \mathbb{R} \rightarrow$ $D \subset \mathbb{R}^{m}$ with $L^{\infty}$ norm smaller than $D_{p}\left(x_{\ell}\right)$, a continuous function $\mathfrak{A}_{\ell, d}: \mathbb{R} \times \mathfrak{O}_{\ell} \rightarrow M$ exists such that the map $\xi \mapsto \mathfrak{A}_{\ell, d}(t, \xi)$ is locally Lipschitz (uniformly in $t$ ) and any solution $X\left(t, x, t_{0} ; d\right)$ defined at least on $\left[t_{0},+\infty\right)$ and for which there exists $s$ such that

$$
X\left(t, x, t_{0} ; d\right) \in \mathcal{P}\left(x_{\ell}\right) \quad \forall t \geq s
$$

necessarily satisfies

$$
\begin{aligned}
& X\left(t, x, t_{0} ; d\right) \in \mathfrak{A}_{\ell, d}\left(t, \mathfrak{O}_{\ell}\right) \cap \mathcal{P}\left(x_{\ell}\right) \quad \forall t \geq s . \\
& \text { Remark 1: }
\end{aligned}
$$

1) In the above statement, $p$ is the dimension of the stable manifold of $x_{\ell}$. When $p=0$ (as it is always the case for $n=1), \mathbb{R}^{0}$ denotes the singleton $\{0\}$.

2) For each $t$ in $\mathbb{R}$, the set $\mathfrak{A}_{\ell, d}\left(t, \mathfrak{O}_{\ell}\right)$ is a $p$ rectifiable set (see [7, 3.2.14] for instance for a definition). Since $p$ is strictly smaller than $n$, it has a zero volume. ${ }^{3}$

3) The set $\mathfrak{A}_{\ell, d}\left(t, \mathfrak{O}_{\ell}\right) \cap \mathcal{P}\left(x_{\ell}\right)$ may be empty, in which case no solution exists which is eventually confined within $\mathcal{P}\left(x_{\ell}\right)$.

\section{Proof of Proposition 1.}

Let

- $\delta$ be the smallest distance between the equilibria $x_{\ell}$ in $\mathfrak{C}$ of the undisturbed system.

$$
\text { - } \quad F=2 \max _{x \in \mathfrak{C}}\left|f_{0}(x)\right|_{M}
$$

where $|\cdot|_{M}$ denotes the Riemannian norm of a vector

\footnotetext{
${ }^{3}$ Simply because, for each fixed $t$, the function $(x, y) \in \mathfrak{O}_{\ell} \times \mathbb{R}^{n-p} \mapsto$ $F(x, y) \doteq \mathfrak{A}_{\ell, d}(t, x) \in M$ is locally Lipschitz, it maps zero Lebesgue measure subsets of $\mathbb{R}^{n}$ into zero volume subsets of $M$. Moreover, $F\left(\mathfrak{O}_{\ell} \times\right.$ $\left.\mathbb{R}^{n-p}\right)=F\left(\mathfrak{O}_{\ell} \times\{0\}\right)$ where the subset $\mathfrak{O}_{\ell} \times\{0\}$ of $\mathbb{R}^{p} \times\{0\}$ has zero Lebesgue measure in $\mathbb{R}^{n}$.
} 
field, i.e., for each $x$ in $M$ where $f_{0}$ is defined, we have :

$$
\left|f_{0}(x)\right|_{M}=\sqrt{f_{0}(x)^{T} g(x) f_{0}(x)},
$$

with $g$ being the Riemannian metric.

If $M$ is a not compact, with our definition of the compact set $\mathfrak{C}$, there exists $\varepsilon_{c}>0$ such that :

$$
\left.L_{f} V\right|_{x, 0} \leq-\varepsilon_{c} \quad \forall x: V(x)=v .
$$

With this, (6) and continuity, we can find a strictly positive real number $D_{c}$ such that we have, for all $d$ with $|d| \leq D_{c}$,

$$
\begin{aligned}
\left.\max _{x: V(x)=v} L_{f} V\right|_{x, d} & \leq 0, \\
& \max _{x \in \mathfrak{C}}|f(x, d)|_{M} \leq F .
\end{aligned}
$$

It follows that $\mathfrak{C}$ is forward invariant for the system (2) for all perturbation $d$ with $L^{\infty}$ norm smaller than $D_{c}$.

If $M$ is compact, we have $\mathfrak{C}=M$. Therefore $\mathfrak{C}$ is trivially forward invariant for all perturbation $d$ in $L_{l o c}^{\infty}$. In this case, the real number $D_{c}$ to be used later on can be chosen arbitrarily large (but fixed) and (7) holds again.

To facilitate our forthcoming analysis, we impose also backward completeness. When $M$ is not compact this can be achieved simply by modifying $f$ outside $\mathfrak{C}$ as :

$$
f_{m}(x, d)=\eta(V(x)) f(x, d)
$$

where $\eta$ is a $\mathcal{C}^{\infty}$ function satisfying :

$$
\begin{aligned}
\eta(w)=1 & \text { if } \quad w \leq v, \\
& \in[0,1] \quad \text { if } \quad v \leq w<v+1, \\
=0 & \text { if } \quad v+1 \leq w .
\end{aligned}
$$

Not to overload our notations in this proof, we forget the subscript $m$ for $f$ and we still denote by $X\left(t, x, t_{0}, d\right)$ the solutions of :

$$
\dot{x}=f_{m}(x(t), d(t)) .
$$

Actually, this modification is used only at the very end in the construction of the set $\mathfrak{B}_{d}\left(t_{0}\right)$. Indeed in the remaining part of this proof, we restrict our attention to $x$ in $\mathfrak{C}, t \geq t_{0}$ and $d$ with $L^{\infty}$ norm smaller than $D_{c}$, so there is no difference on $\left[t_{0},+\infty\right)$ between solutions of (2) and solutions of (8).

Let $B_{r}(x)$ and $S_{r}(x)$ denote respectively the Riemannian ball and sphere centered at $x$ and with radius $r$. Let also :

- $r_{e} \leq \frac{\delta}{4}, D_{e}$ and $\varepsilon_{m}$ be strictly positive real numbers such that

1) we have :

$$
\varepsilon_{m}=-\left.\frac{1}{2} \min _{x \in \mathfrak{C} \backslash \cup_{\ell} B \frac{r_{e}}{2}\left(x_{\ell}\right)} L_{f} V\right|_{x, 0} .
$$

2) moreover, for all $d,|d| \leq D_{e}$

$$
\left.\max _{x \in \mathfrak{C} \backslash \cup_{\ell} B_{\frac{r_{e}}{2}}\left(x_{\ell}\right)} L_{f} V\right|_{x, d} \leq-\varepsilon_{m} .
$$

3) finally, for each equilibrium $x_{\ell}$ of the undisturbed system which is not asymptotically stable it holds

$$
B_{r_{e}}\left(x_{\ell}\right) \subset \mathcal{P}\left(x_{\ell}\right), \quad D_{e} \leq D_{p}\left(x_{\ell}\right)
$$

where $\mathcal{P}\left(x_{\ell}\right)$ and $D_{p}\left(x_{\ell}\right)$ are respectively the set and the real number given by Lemma 1 .

- $\mathcal{Q}\left(x_{\ell}\right) \subset B_{\frac{r_{e}}{2}}\left(x_{\ell}\right)$ be a neighborhood of $x_{\ell}$ defined as follows :

- If $x_{\ell}$ is asymptotically stable, $V$ being strictly decreasing along solutions of the undisturbed system, $x_{\ell}$ is a strict local minimum of $V$. Thus, together with (4), continuity and compactness imply the existence of a compact neighborhood $\mathcal{Q}\left(x_{\ell}\right)$ of $x_{\ell}$ which is a connected component of a sublevel set of $V$ and a subset of $B_{\frac{r_{e}}{2}}\left(x_{\ell}\right)$, and strictly positive real numbers $D_{i}\left(x_{\ell}\right) \varepsilon_{i}\left(x_{\ell}\right)$ so that for all pairs $(x, d)$ with $x \in B_{\frac{r_{\ell}}{2}}\left(x_{\ell}\right) \backslash \mathcal{Q}\left(x_{\ell}\right)$ and $|d| \leq D_{i}\left(x_{\ell}\right)$ it holds:

$$
\left.L_{f} V\right|_{x, d}<-\varepsilon_{i}\left(x_{\ell}\right) .
$$

- If $x_{\ell}$ is not asymptotically stable, we pick $r_{i}\left(x_{\ell}\right)$ in $\left(0, \frac{r_{e}}{2}\right)$ so that, by letting

$$
\mathcal{Q}\left(x_{\ell}\right)=B_{r_{i}\left(x_{\ell}\right)}\left(x_{\ell}\right),
$$

we have :

$$
\max _{x \in \mathcal{Q}\left(x_{\ell}\right)} V(x)-\min _{x \in \mathcal{Q}\left(x_{\ell}\right)} V(x)<\varepsilon_{m} \frac{r_{e}}{F}
$$

The continuity of $V$ guarantees that such an $r_{i}\left(x_{\ell}\right)$ exists. Then again, continuity and compactness imply the existence of strictly positive real numbers $D_{i}\left(x_{\ell}\right)$ and $\varepsilon_{i}\left(x_{\ell}\right)$ so that (11) holds.

With all these definitions, we have :

$$
\left.L_{f} V\right|_{x, d}<-\min \left\{\varepsilon_{m}, \varepsilon_{i}\left(x_{\ell}\right)\right\}<0
$$

for all pairs $(x, d)$ with $x \in \mathfrak{C} \backslash \bigcup_{\ell} \mathcal{Q}\left(x_{\ell}\right)$ and $|d| \leq$ $\min \left\{D_{e}, D_{c}, \min _{\ell} D_{i}\left(x_{\ell}\right)\right\}$.

Also any solution which leaves a ball $B \frac{r_{e}}{2}\left(x_{\ell}\right)$ and reaches a sphere $S_{\frac{r_{e}}{2}}\left(x_{j}\right)$, with $j \neq \ell$, must "travel" during a time which is at least $\frac{\delta-r_{e}}{F}$. And during this time the Lyapunov function decreases by an amount which is at least

$$
\Delta=\varepsilon_{m} \frac{\delta-r_{e}}{F} \geq 4 \varepsilon_{m} \frac{r_{e}}{F} \text {. }
$$

From now on, we restrict our attention to perturbations with $L^{\infty}$ norm smaller than $\min \left\{D_{e}, D_{c}, \min _{\ell} D_{i}\left(x_{\ell}\right)\right\}$.

Pick a solution $X\left(t, x, t_{0} ; d\right)$ which at time say $t_{C}$ is in $\mathfrak{C}$. This compact set being forward invariant, the solution is in it for all times $t \geq t_{C}$. Since $V$ is lower bounded, we conclude from (13), that this solution must enter or start from one of the sets $\mathcal{Q}\left(x_{\ell}\right)$ and, furthermore, can only spend finite time intervals outside of $\bigcup_{\ell} \mathcal{Q}\left(x_{\ell}\right)$.

In the following, we shall prove

Claim 1: There exists a time $t_{*}$ and an index $\ell_{*}$ such that we have :

$$
X\left(t, x, t_{0} ; d\right) \in \mathcal{Q}\left(x_{\ell_{*}}\right) \quad \forall t \geq t_{*} .
$$

Assuming for the time being this claim holds true, then

either $x_{\ell_{*}}$ is asymptotically stable. In this case, local 
asymptotic stability implies local Input-to-State Stability (ISS), for suitable restrictions on inputs and initial conditions. This result is usually stated for systems defined on Euclidean space (see Lemma I.1 in [14]), however, due to its local nature, it can be adapted straightforwardly to systems on manifolds. The estimate in equation (5) is a direct consequence of local ISS.

or, $x_{\ell_{*}}$ is not asymptotically stable. In this case, then since (see (10))

$$
\mathcal{Q}\left(x_{\ell_{*}}\right) \subset B_{r_{e}}\left(x_{\ell_{*}}\right) \subset \mathcal{P}\left(x_{\ell_{*}}\right),
$$

with Lemma 1 , the solution $t \mapsto X\left(t, x, t_{0} ; d\right)$ is in $\mathfrak{A}_{\ell_{*}, d}\left(t, \mathfrak{O}_{\ell_{*}}\right) \cap B_{r_{e}}\left(x_{\ell_{*}}\right)$ for each $t \geq t_{*}$ and therefore also in $\mathfrak{A}_{\ell_{*}, d}\left(i, \mathfrak{O}_{\ell_{*}}\right) \cap B_{r_{e}}\left(x_{\ell_{*}}\right)$ for each integer $i$ larger or equal to $t_{*}$. But this says that at time $t_{0}$ the solution was at $x$ which is in ${ }^{4}$ :

$$
\mathfrak{C} \bigcap \bigcup_{i \in \mathbb{N} \geq t_{0}} X\left(t_{0}, \mathfrak{A}_{\ell_{*}, d}\left(i, \mathfrak{O}_{\ell_{*}}\right), i ; d\right)
$$

and therefore in the set $\mathfrak{B}_{d}\left(t_{0}\right)$ defined as :

$$
\mathfrak{B}_{d}\left(t_{0}\right)=\mathfrak{C} \bigcap \bigcup_{\ell \leq \mathfrak{L}, i \in \mathbb{N} \geq t_{0}} X\left(t_{0}, \mathfrak{A}_{\ell, d}\left(i, \mathfrak{O}_{\ell}\right), i ; d\right) .
$$

In other words, if $x$ is not in $\mathfrak{B}_{d}\left(t_{0}\right)$ then the set $\mathcal{Q}\left(x_{\ell_{*}}\right)$ in which the solution $X\left(t, x, t_{0} ; d\right)$ ends must be associated to an equilibrium $x_{\ell_{*}}$ which is asymptotically stable. Note finally that, since for any given pair $\left(t_{0}, i\right)$, the function

$$
\left.x \mapsto X\left(t_{0}, x, i ; d\right)\right)
$$

is Lipschitz on $\mathfrak{C}, \mathfrak{B}_{d}\left(t_{0}\right)$ is a countable union of images by Lipschitz maps of $p$-rectifiable sets, with $p \leq n-1$ and therefore is countably $n-1$-rectifiable. So it has zero volume.

To complete our proof, it remains to prove claim 1 . Since the solution must enter one of the sets $\mathcal{Q}\left(x_{\ell}\right)$, say at time $s$,

either $x_{\ell}$ is asymptotically stable, then $\mathcal{Q}\left(x_{\ell}\right)$ is a connected component of a sublevel set of $V$ which, with (11), is (strictly) forward invariant. So the solution will never leave it in future times.

or $x_{\ell}$ is not asymptotically stable; in this case if the solution $t \mapsto X\left(t, x, t_{0} ; d\right)$, which is in the interior of $B_{r_{e}}\left(x_{\ell}\right)$ at time $s$, reaches $S_{r_{e}}\left(x_{\ell}\right)$ at time $\tau>s$, it will never again enter $\mathcal{Q}\left(x_{\ell}\right)$, as established below.

Note that since the solution can only spend finite time intervals outside $\bigcup_{\ell} \mathcal{Q}\left(x_{\ell}\right)$ and the number of $x_{\ell}$ is finite, this proves the claim. So for the sake of getting a contradiction, assume the solution does re-enter $\mathcal{Q}\left(x_{\ell}\right)$ at a time $\bar{\tau}>\tau>s$. Two sub-cases are possible.

Case 2.1 : Meanwhile it did not enter any other set $\mathcal{Q}\left(x_{k}\right)$. In this case the Lyapunov function has been decreasing whenever the solution was not in $\mathcal{Q}\left(x_{\ell}\right)$. Then because we

\footnotetext{
${ }^{4}$ Recall that in this proof $X\left(t, x, t_{0}\right)$ is a solution of (8), system which by construction is backward complete.
}

have $X\left(s, x, t_{0} ; d\right) \in \mathcal{Q}\left(x_{\ell}\right), X\left(\tau, x, t_{0} ; d\right) \in S_{r_{e}}\left(x_{\ell}\right)$ and $X\left(\bar{\tau}, x, t_{0} ; d\right) \in \partial \mathcal{Q}\left(x_{\ell}\right)$ there exist

such that we have :

$$
\tau_{1} \leq \tau_{2}<\tau \leq \tau_{3}<\tau_{4} \leq \bar{\tau}
$$

$$
\begin{aligned}
& X\left(\tau_{1}, x, t_{0} ; d\right) \in \partial \mathcal{Q}\left(x_{\ell}\right) \\
& \mathfrak{d}_{M}\left(x_{\ell}, X\left(\tau_{2}, x, t_{0} ; d\right)\right)=\frac{r_{e}}{2} \\
& \mathfrak{d}_{M}\left(x_{\ell}, X\left(\tau, x, t_{0} ; d\right)\right)=r_{e}, \\
& \mathfrak{d}_{M}\left(x_{\ell}, X\left(\tau_{3}, x, t_{0} ; d\right)\right)=r_{e}, \\
& \mathfrak{d}_{M}\left(x_{\ell}, X\left(\tau_{4}, x, t_{0} ; d\right)\right)=\frac{r_{e}}{2} .
\end{aligned}
$$

Specifically

- at time $\tau_{1}$, the solution is in the boundary of $\mathcal{Q}\left(x_{\ell}\right)$ and, on the time interval $\left(\tau_{1}, \bar{\tau}\right)$, the solution is not in $\mathcal{Q}\left(x_{\ell}\right)$. This implies that on this time interval the Lyapunov function decreases.

- the interval $\left(\tau_{2}, \tau\right]$ is defined so that the solution is in $B_{r_{e}}\left(x_{\ell}\right) \backslash B_{\frac{r_{e}}{2}}\left(x_{\ell}\right)$ while it belongs to the sphere $S_{\frac{r_{e}}{2}}\left(x_{\ell}\right)$ at time $\tau_{2}$ and to the sphere $S_{r_{e}}\left(x_{\ell}\right)$ at time $\tau$.

- the interval $\left[\tau_{3}, \tau_{4}\right.$ ) (with $\tau_{3} \geq \tau$ ) is defined so that the solution is back to $B_{r_{e}}\left(x_{\ell}\right) \backslash B r_{\frac{r_{e}}{2}}\left(x_{\ell}\right)$, being in the sphere $S_{r_{e}}\left(x_{\ell}\right)$ at time $\tau_{3}$ and in the sphere $S_{\frac{r_{e}}{2}}\left(x_{\ell}\right)$ at time $\tau_{4}$.

The above considerations and definitions imply:

- between $\tau_{1}$ and $\tau_{2}$, the solution is not in $\mathcal{Q}\left(x_{\ell}\right)$. So $V$ decreases but we do not estimate by how much.

- between $\tau_{2}$ and $\tau$, the solution is in $B_{r_{e}}\left(x_{\ell}\right) \backslash B_{\frac{r_{e}}{2}}\left(x_{\ell}\right)$ and $V$ decreases by at least $\varepsilon_{m}\left(\tau-\tau_{2}\right)$ which is lower bounded by $\varepsilon_{m} \frac{r_{e}}{2 F}$.

- between $\tau$ and $\tau_{3}, V$ continues to decrease.

- between $\tau_{3}$ and $\tau_{4}$ the solution is in $B_{r_{e}}\left(x_{\ell}\right) \backslash B_{\frac{r_{e}}{2}}\left(x_{\ell}\right)$ and $V$ decreases at least by $\varepsilon_{m}\left(\tau_{4}-\tau_{3}\right)$ which is again lower bounded by $\varepsilon_{m} \frac{r_{e}}{2 F}$.

- finally on the interval $\left(\tau_{4}, \bar{\tau}\right)$, the solution is not in $\mathcal{Q}\left(x_{\ell}\right)$ so $V$ is still decreasing.

Using (6) and (7), we have :

$\mathfrak{d}_{M}\left(x_{\ell}, X\left(\tau, x, t_{0} ; d\right)\right) \leq$

$\mathfrak{d}_{M}\left(x_{\ell}, X\left(\tau_{2}, x, t_{0} ; d\right)\right)+\mathfrak{d}_{M}\left(X\left(\tau, x, t_{0} ; d\right), X\left(\tau_{2}, x, t_{0} ; d\right)\right)$

$\mathfrak{d}_{M}\left(X\left(\tau, x, t_{0} ; d\right), X\left(\tau_{2}, x, t_{0} ; d\right)\right)$

$$
\begin{array}{r}
\leq \int_{\tau_{2}}^{\tau}\left|f\left(X\left(r, x, t_{0} ; d\right), d(r)\right)\right|_{M} d r \\
\leq F\left(\tau-\tau_{2}\right)
\end{array}
$$

This yields :

$$
\begin{aligned}
r_{e} & =\mathfrak{d}_{M}\left(x_{\ell}, X\left(\tau, x, t_{0} ; d\right)\right) \\
& \leq \mathfrak{d}_{M}\left(x_{\ell}, X\left(\tau_{2}, x, t_{0} ; d\right)\right)+F\left[\tau-\tau_{2}\right] \\
& \leq \frac{r_{e}}{2}+F\left[\tau-\tau_{2}\right] .
\end{aligned}
$$

Similarly, we have :

$$
\begin{aligned}
\frac{r_{e}}{2} & =\mathfrak{d}_{M}\left(x_{\ell}, X\left(\tau_{4}, x, t_{0} ; d\right)\right) \\
& \geq \mathfrak{d}_{M}\left(x_{\ell}, X\left(\tau, x, t_{0} ; d\right)\right)-F\left[\tau_{4}-\tau\right] \\
& \geq r_{e}-F\left[\tau_{4}-\tau\right]
\end{aligned}
$$

and therefore :

$$
r_{e} \leq F\left[\tau_{4}-\tau_{2}\right]
$$


So, with (9) and (11), we get :

$$
\begin{aligned}
\min _{x \in \mathcal{Q}\left(x_{\ell}\right)} V(x) & \leq V\left(X\left(\bar{\tau}, x, t_{0} ; d\right)\right) \leq V\left(X\left(\tau_{4}, x, t_{0} ; d\right)\right) \\
& \leq V\left(X\left(\tau_{2}, x, t_{0} ; d\right)\right)-\varepsilon_{m}\left[\tau_{4}-\tau_{2}\right] \\
& \leq V\left(X\left(\tau_{2}, x, t_{0} ; d\right)\right)-\varepsilon_{m} \frac{r_{e}}{F} \\
& \leq V\left(X\left(\tau_{1}, x, t_{0} ; d\right)\right)-\varepsilon_{m} \frac{r_{e}}{F} \\
& \leq \max _{x \in \mathcal{Q}\left(x_{\ell}\right)} V(x)-\varepsilon_{m} \frac{r_{e}}{F} .
\end{aligned}
$$

This contradicts (12). So, at least in this case, the solution cannot re-enter $\mathcal{Q}\left(x_{\ell}\right)$.

Case $2.2:$ Meanwhile it has entered at least another set $\mathcal{Q}\left(x_{k}\right)$, with $x_{k} \neq x_{\ell}$. The analysis can be carried out by means of similar techniques and for space reasons we do not include it here.

\section{A SUFFICIENT CONDITION FOR ALMOST GLOBAL INPUT-TO-STATE STABILITY}

The Main result in the previous Section will be used in order to develop a checkable sufficient condition for the notion of almost global Input-to-State Stability (aISS), recently introduced in [1], which we recall below:

Definition 1: A system as in (2) is said to be almost globally Input-to-State Stable with respect to a compact subset $A \subset M$ if $A$ is locally asymptotically stable for $d \equiv 0$ and there exists $\tilde{\gamma} \in \mathcal{K}$ such that for each locally essentially bounded and measurable perturbation $d: \mathbb{R} \rightarrow \mathcal{D}$, there exists a zero volume set $\tilde{\mathfrak{B}}_{d} \subset M$ such that, for all $x \in M \backslash \tilde{\mathfrak{B}}_{d}$, it holds:

$$
\limsup _{t \rightarrow+\infty} \mathfrak{d}_{M}(X(t, x, 0 ; d), A) \leq \tilde{\gamma}\left(\|d\|_{\infty}\right) .
$$

Notice that in this last inequality we specify $t_{0}=0$, without loss of generality.

Remark 2: This notion is useful in many different contexts, both for systems with $M=\mathbb{R}^{n}$ (for instance when $A$ is a limit cycle or a set of more than one equilibrium point), as well as for nonlinear systems evolving on manifolds non diffeomorphic to Euclidean space (in which case even $A$ being a single equilibrium requires almost global tools to be handled). Despite its potential interest, few sufficient conditions are available to prove this holds in actual examples. It is also worth pointing out that it is a purely open-loop notion of robustness; as $\tilde{\mathfrak{B}}_{d}$ is $d$ dependent, letting $d$ be a function of $x$ is generally not possible.

Definition 2: A system as in (2) fulfills the Ultimate Boundedness property if there exists a class $\mathcal{K}$ function $\delta$, a constant $c$ and a point $\xi \in M$ such that for each $x \in M$, and each locally essentially bounded and measurable perturbation $d$, the solution $X(t, x, 0 ; d)$ is defined on $[0,+\infty)$, and

$$
\lim _{t \rightarrow+\infty} \mathfrak{d}_{M}\left(X(t, x, 0 ; d), S_{\text {res }}\right)=0
$$

where $S_{\text {res }}$ is the set $\left\{z \in M: \mathfrak{d}_{M}(z, \xi) \leq \delta\left(\|d\|_{\infty}\right)+c\right\}$.

We remark that in the above definition Ultimate Boundedness could have been equivalently defined by considering the point-set distance to a compact subset of $M$, rather than a singleton $\{\xi\}$. Our main result for this Section is stated below. Also, of course, the Ultimate Boundedness property holds always when $M$ is compact.

Proposition 2: Consider a system as in (2) which fulfills all the assumptions of Proposition 1. Assume, in addition, that the set of asymptotically stable equilibria of (3) be finite and be denoted by $E_{s}$. If Ultimate Boundedness holds, then, (2) is almost globally ISS with respect to the set $E_{s}$.

Proof. By Ultimate Boundedness there exist a function $\tilde{\delta}$ of class $\mathcal{K}$ and a constant $\tilde{c}$ such that for each $x \in M$, and each locally essentially bounded and measurable perturbation $d$, the solution $X(t, x, 0 ; d)$ is defined on $[0,+\infty)$ and fulfills :

$$
\limsup _{t \rightarrow+\infty} \mathfrak{d}_{M}\left(X(t, x, 0 ; d), E_{s}\right) \leq \tilde{c}+\tilde{\delta}\left(\|d\|_{\infty}\right) .
$$

Let the compact set $\mathfrak{C}$ invoked in the main result be selected to contain the set $\left\{x \in M: \mathfrak{d}_{M}\left(x, E_{s}\right) \leq \tilde{c}+\tilde{\delta}(1)+1\right\}$. Then, let $\Delta>0$ be given as from our main result. Fix $d$, as an arbitrary measurable perturbation which is essentially bounded (for unbounded $d$ there is nothing to prove). Since a Riemannian manifold is $\sigma$-compact ${ }^{5}$, we can pick a monotone increasing sequence of compact subsets of $M$, $K_{1} \subset K_{2} \subset \ldots \subset K_{n} \subset \ldots$ with the property that $\bigcup_{n \in \mathbb{N}} K_{n}=M$. Assume $\left.\|d\|_{\infty} \leq \min \{\Delta, 1)\right\}$. By virtue of (15), and continuity of solutions with respect to initial conditions, for all $n \in \mathbb{N}$ there exists $T_{n}>0$ such that $X\left(T_{n}, K_{n}, 0 ; d\right)$ is a subset of $\mathfrak{C}$. Then, applying our main result yields existence of a zero volume set $\mathfrak{B}_{d n} \subset \mathfrak{C}$ such that, for all $x \in K_{n}$ such that $X\left(T_{n}, x, 0 ; d\right)$ is in $\mathfrak{C} \backslash \mathfrak{B}_{n}$, it holds

$$
\limsup _{t \rightarrow+\infty} \mathfrak{d}_{M}\left(X(t, x, 0 ; d), E_{s}\right) \leq \gamma\left(\|d\|_{\infty}\right) .
$$

Since $x \mapsto X\left(T_{n}, x, 0 ; d\right)$ is a diffeomorphism which preserves zero volume sets and with inverse $\chi \mapsto$ $X\left(0, \chi, T_{n} ; d\right)$, it follows that $\tilde{\mathfrak{B}}_{d n}=X\left(0, \mathfrak{B}_{n}, T_{n} ; d\right)$ has zero volume and, for each $\tilde{x} \in K_{n} \backslash \tilde{\mathfrak{B}}_{d n}$ (16) holds.

Let $\tilde{\mathfrak{B}}:=\bigcup_{n \in \mathbb{N}} \tilde{\mathfrak{B}}_{d n}$. It has zero volume as a countable union of zero volume sets. Moreover, for all $x \in M \backslash \tilde{\mathfrak{B}}$ there exists $n \in \mathbb{N}$ so that $x \in K_{n}$; thus $x \in K_{n} \backslash \tilde{\mathfrak{B}} \subset$ $K_{n} \backslash \tilde{\mathfrak{B}}_{d n}$ and inequality in (16) holds. Finally, almost global ISS follows simply by combining (16) and condition (15) with $\tilde{\gamma}$ given as :

$$
\begin{aligned}
& \tilde{\gamma}(s)=\gamma(s)_{\tilde{\delta}} \quad \text { if } \quad s \leq \min \{\Delta, 1\}, \\
& \geq \tilde{c}+\tilde{\delta}(s) \text { if } \min \{\Delta, 1\}<s .
\end{aligned}
$$

Proposition 3: Consider a system as in (2), and assume there exist a $\mathcal{K}_{\infty}$ function $\alpha$ and a $\mathcal{C}^{1}$ and proper function $W: M \rightarrow \mathbb{R}_{\geq 0}$ satisfying:

$$
\left.L_{f} W\right|_{x, d} \leq-\alpha(W(x))+c+\delta(|d|)
$$

for all $x \in M$ and all $d \in \mathcal{D}$. Then, system (2) fulfills the Ultimate Boundedness property.

Proof. By virtue of (17), it holds:

\footnotetext{
${ }^{5}$ A Riemannian manifold is locally compact (see [5, Theorem VI.6.6 and page 335]) and paracompact (Stone Theorem) (see [17, Theorem 20.9]). Moreover, a paracompact, locally compact and connected space is $\sigma$-compact (see [8, Lemmas 5 and 6] ).
} 


$$
\limsup _{t \rightarrow+\infty} \alpha(W(x(t))) \leq 2\left(c+\delta\left(\mid d \|_{\infty}\right)\right) .
$$

As $W$ is proper, taken any $z \in M, \kappa$ of class $\mathcal{K}_{\infty}$ and a constant $c_{z}$ exist, so that for all $x \in M$

$$
\kappa\left(\mathfrak{d}_{M}(z, x)\right) \leq W(x)+c_{z} .
$$

Combining (19) and (18) proves Ultimate Boundedness.

\section{A. A planar example: pendulum with friction}

Consider the following set of differential equations, describing the motion of a forced pendulum with friction:

$$
\begin{aligned}
\dot{\theta} & =\omega \\
\dot{\omega} & =-a \sin (\theta)-b \omega+d .
\end{aligned}
$$

We regard them as a system with state $x=[\theta, \omega]$ taking values on the cylinder $M:=\mathbb{S} \times \mathbb{R}$ affected by some exogenous disturbance $d(t)$, whereas $a, b$ are constant positive parameters. The following question was publicly posed in Oberwolfach meeting: is the above system almost globally Input-to-State Stable ? Consider the mechanical energy of the pendulum, that is $W(x)=\omega^{2} / 2-a \cos (\theta)$. Taking derivatives along (20) yields:

$$
\begin{aligned}
\dot{W}(x) & =-b \omega^{2}+\omega d \leq-\frac{b}{2} \omega^{2}+\frac{1}{2 b} d^{2} \\
& =-\frac{b}{2} W(x)-\frac{a b}{2} \cos (\theta)+\frac{1}{2 b} d^{2} \\
& \leq-\frac{b}{2} W(x)+c+\frac{1}{2 b} d^{2}
\end{aligned}
$$

with constant $c:=a b / 2$. By virtue of (17), system (20) fulfills Ultimate Boundedness. Moreover, it is straightforward to see that (20) has only two equilibria $x_{1}=[0,0]$ and $x_{2}=[ \pm \pi, 0]$. In particular, $x_{1}$ is asymptotically stable, whereas $x_{2}$ is an hyperbolic saddle point. Let us denote $X_{e}:=\left\{x_{1}, x_{2}\right\}$. In order to build a strict Lyapunov function for (20) we perturb $W$ as follows:

$$
V(x)=W(x)+\varepsilon \omega \sin (\theta)
$$

for some small parameter $\varepsilon$ to be fixed later. Along solutions of the autonomous system $V$ fulfills the following dissipation inequality:

$$
\begin{aligned}
\dot{V}(x) & =d V(x) \cdot f(x, 0) \\
& =-b \omega^{2}-\varepsilon a \sin ^{2}(\theta)-\varepsilon b \omega \sin (\theta)+\varepsilon \omega^{2} \cos (\theta) \\
& \leq-(b-\varepsilon) \omega^{2}-\varepsilon a \sin ^{2}(\theta)-\varepsilon b \omega \sin (\theta)<0
\end{aligned}
$$

for all $x \notin X_{e}$, provided $b>\varepsilon$ and $a(b-\varepsilon)>\varepsilon b^{2} / 4$. The previous inequalities can be simultaneously fulfilled by taking $\varepsilon$ sufficiently small. Hence, the pendulum equations fulfill all assumptions of our previous result, and we can therefore conclude almost global Input-to-State Stability.

\section{B. A scalar counter-example}

We show next, by means of a simple scalar example, that the existence of at least one unstable eigenvalue is an assumption which cannot be removed from the Main Result.

Let $M=\mathbb{S}$ and $\theta$ be the corresponding angular coordinate on $\mathbb{S}$. Consider the system:

$$
\dot{\theta}=-\sin ^{3}(\theta)+\sin (\theta) d
$$

For $d=0$ the system has two equilibria, namely $\theta=0$ which is asymptotically stable and $\theta=\pi$ which is antistable. Notice that $d f_{0}(\pi)=0$, so that the linearized system does not have positive eigenvalues at the unstable equilibrium. We want to show that, even for arbitrarily small input signals it is not true that almost all solutions converge to a neighborhood of 0 whose volume shrinks to 0 as the input perturbation $L_{\infty}$ norm tends to 0 . Indeed, taking constant inputs we obtain $\left.d f(\theta, d)\right|_{\theta=\pi}=-d$ which yields the linearized system:

$$
\delta \dot{\theta}=-d \delta \theta \text {. }
$$

Therefore, for all $d>0$ we have local asymptotic stability of the equilibrium at $\theta=\pi$. This proves that, no matter how small we pick $d$ there always exists a basin of attraction of positive measure for the equilibrium $\theta=\pi$. This simple example justifies our assumption on $d f_{0}$.

\section{REFERENCES}

[1] D. Angeli, "An almost global notion of Input-to-State Stability", IEEE Trans. on Automatic Control, Vol. 49, pp. 866-874, 2004.

[2] D. Angeli, "Some remarks on density functions for dual Lyapunov methods", Proc. of the 42 IEEE Conference on Decision and Control, pp. 5080-5082, 2003.

[3] D. Angeli, "Open problem: almost Input-to-State Stability", Mathematisches Forschungsinstitut Oberwolfach Report No. 11, pp. 670-671, 2009.

[4] B. Aulbach and T. Wanner, "Integral Manifolds for Caratheodory Type Differential Equations in Banach Spaces", in Six Lectures on Dynamical Systems, edited by B. Aulbach and F. Colonius. World Scientific, 1996.

[5] W. M. Boothby, An introduction to differentiable manifolds and Riemannian geometry, Academic Press, 1975.

[6] C.A. Desoer and M. Vidyasagar, Feedback Systems : Input-Output Properties, SIAM, 2008.

[7] H. Federer, Geometric Measure Theory, Classics in Math., Vol. XVI, Springer-Verlag, New York, 1996.

[8] D.B. Gauld, "Topological Properties of Manifolds", The American Mathematical Monthly, Vol. 81 (6), pp: 633-636, 1974.

[9] S. Prajna, P.A Parrilo, and A. Rantzer, "Nonlinear Control Synthesis by Convex Optimization", IEEE Trans. on Automatic Control, Vol. 49, N. 2, pp. 310-314, 2004.

[10] S. Prajna and A. Rantzer, "On Homogeneous Density Functions", in Anders Rantzer, C. I. Byrnes (Eds.): Directions in Mathematical Systems Theory and Control, Springer Verlag, Berlin Heidelberg, 2003.

[11] A. Rantzer, "A dual to Lyapunov's stability theorem", Systems and Control Letters, Vol. 42, pp. 161-168, 2001.

[12] A. Rantzer, "An converse theorem for density functions", Proc. of the 41st IEEE Conf. on Decision and Control, pp. 1890-1891, Las Vegas, 2002.

[13] E.D. Sontag, "Input to state stability: Basic concepts and results", in P. Nistri and G. Stefani, editors, Nonlinear and Optimal Control Theory, Springer-Verlag, Berlin, pp. 163-220, 2007.

[14] E. Sontag and Y. Wang, "New characterizations of Input-to-State Stability", IEEE Trans. on Automatic Control, Vol. 41, N. 9, pp. 12831294, 1996.

[15] A.R. Teel and L. Praly, "A smooth Lyapunov function from a class-KL estimate involving two positive semidefinite functions", Esaim-Control Optimisation and Calculus of Variations, Vol. 5, pp. 313-367, 2000.

[16] A. van der Schaft, $L_{2}$-gain and Passivity Techniques in Nonlinear Control, Communications and Control Engineering Series, Springer, Berlin, 1999.

[17] S. Willard, General Topology, Addison-Wesley, Massachusetts, 1970, Published by Dover (2004).

Acknowledgements: The authors wish to thank Prof. JeanMichel Coron for pointing out reference [8]. The first author wishes to thank Prof. Rantzer and Prof. Sepulchre for useful discussions on the subject of this note. 